\title{
Aberrant Loss of Surface CD3 and TCR $\gamma \delta$ Expression in Relapsed Hepatosplenic T-cell Lymphoma
}

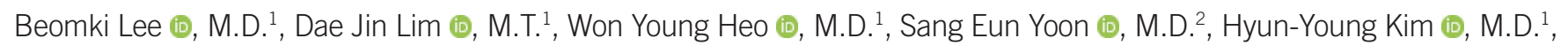

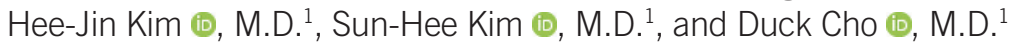

${ }^{1}$ Department of Laboratory Medicine and Genetics, Samsung Medical Center, Sungkyunkwan University School of Medicine, Seoul, Korea; ${ }^{2}$ Division of Hematology-Oncology, Department of Medicine, Samsung Medical Center, Sungkyunkwan University School of Medicine, Seoul, Korea

\section{Dear Editor,}

Hepatosplenic T-cell lymphoma (HSTL) is an aggressive extranodal lymphoma that originates from cytotoxic $\gamma \delta$ T cells $[1,2]$. HSTL patients typically present with hepatosplenomegaly in the absence of lymphadenopathy [1, 2]. Morphologically, HSTL is characterized by sinusoidal infiltration of the spleen, liver, and bone marrow (BM) with monotonous medium-sized neoplastic lymphoid cells; isochromosome $7 q$ is a recurrent cytogenetic abnormality detected in most HSTL cases [1, 2]. The typical immunophenotype of HSTL is as follows: CD2+, SCD3+, CD4-, CD5-, CD7+, CD8-, and T-cell receptor (TCR) $\gamma \delta+[1]$. However, there have been reports of rare HSTL cases with loss of SCD3 and TCR $\gamma \delta$, posing a major challenge in establishing an accurate diagnosis and determining relapse [3-6]. We report a rare case of HSTL with an unusual immunophenotype characterized by loss of SCD3 and TCR $\gamma \delta$ expression at relapse. Unlike previously reported cases, the abnormal cells in our case were positive for CD16 and CD56 and, therefore, could be easily misinterpreted as natural killer (NK) cells. The Institutional Review Board of Samsung Medical Center, Seoul, Korea, approved this study (approval number: 2020-07-154) and waived the need for informed consent.

\section{A 57-year-old man visited Samsung Medical Center in Sep-}

tember 2019 for fever of unknown origin (FUO). The complete blood count $(\mathrm{CBC})$ result was as follows: hemoglobin, $81 \mathrm{~g} / \mathrm{L}$; white blood cell count, $3.38 \times 10^{9} / \mathrm{L}$ (without definite abnormal lymphoid cells); and platelet count, $204 \times 10^{9} / \mathrm{L}$. The abdominopelvic computed tomography scan revealed hepatosplenomegaly, with a spleen size of $16 \mathrm{~cm}$. During the FUO workup, a BM biopsy was performed, which led to the diagnosis of mature $\gamma \delta$ T-cell lymphoma without an identifiable primary focus. In the initial study, the BM aspirate showed 5-10\% of medium-sized abnormal lymphoid cells with pleomorphic nuclei and prominent nucleoli. Flow cytometry was performed using a FACSLyric flow cytometer (Becton Dickinson, San Jose, CA, USA) after staining the $\mathrm{BM}$ mononuclear cells with monoclonal antibodies against T- and NK-cell associated antigens, and data were analyzed using the Kaluza software (Beckman Coulter Inc., Miami, FL, USA). Flow cytometry revealed an abnormal T-cell population with the following immunophenotype: SCD3+, cCD3+, CD4-, CD8-, CD56+, CD16+, CD2+, CD5-, CD7+, CD94+, CD57-, and TCR $\gamma \delta+(F i g$. 1). Chromosomal analysis indicated a normal karyotype (46,XY[20]), and a TCR rearrangement assay revealed clonality in both $T C R \gamma$ and TCR $\beta$.

The patient underwent multiple cycles of chemotherapy with four different regimens. During the course of chemotherapy, sple-
Received: September 18, 2020

Revision received: December 1, 2020

Accepted: May 7, 2021

Corresponding author: Duck Cho, M.D.

Department of Laboratory Medicine and Genetics, Samsung Medical Center, Sungkyunkwan University School of Medicine, 81 Irwon-ro, Gangnam-gu, Seoul 06351, Korea

Tel: +82-2-3410-2403, Fax: +82-2-3410-2719

E-mail: duck.cho@skku.edu 
nectomy and cholecystectomy were performed, and spleen pathology revealed $\gamma \delta$ T-cell lymphoma. A follow-up BM study was conducted six months after the initial diagnosis, and the CBC result was as follows: hemoglobin, $105 \mathrm{~g} / \mathrm{L}$; white blood cell count, $10.22 \times 10^{9} / \mathrm{L}$ (10\% abnormal lymphoid cells); and platelet count, $244 \times 10 \%$ L. The BM aspirate showed $20 \%$ abnormal lymphoid cells, and their immunophenotype, determined by flow cytometry, was sCD3-, cCD3+, CD4-, CD8-, CD56+, CD16+, CD2+, CD5-, CD7+, CD94+, CD57-, and TCR $\gamma \delta-$ (Fig. 1); in contrast to the initial findings, loss of $\mathrm{SCD} 3$ and $\mathrm{TCR} \gamma \delta$ was noted. Two possibilities were considered: 1) an unusual immunophenotypic shift to abnormal T cells lacking sCD3 and TCR $\gamma \delta$ and 2) an increased proportion of reactive NK cells. A positive TCR rearrangement result supported the former possibility; next-generation sequencing (NGS) was not performed. Furthermore, chromosomal analysis revealed a complex karyotype, including isochromosome 7q (46,XY,add(4)(p16),+6,i(7)(q10),der(10;14)(q10;q10),del(14) (q32)[9]/46,idem,add(X)(p21),-add(4),+4[7]/46,XY[4]), and a specific diagnosis of HSTL was finally made. Although the role of isochromosome 7q in HSTL remains unclear, it has been sug-
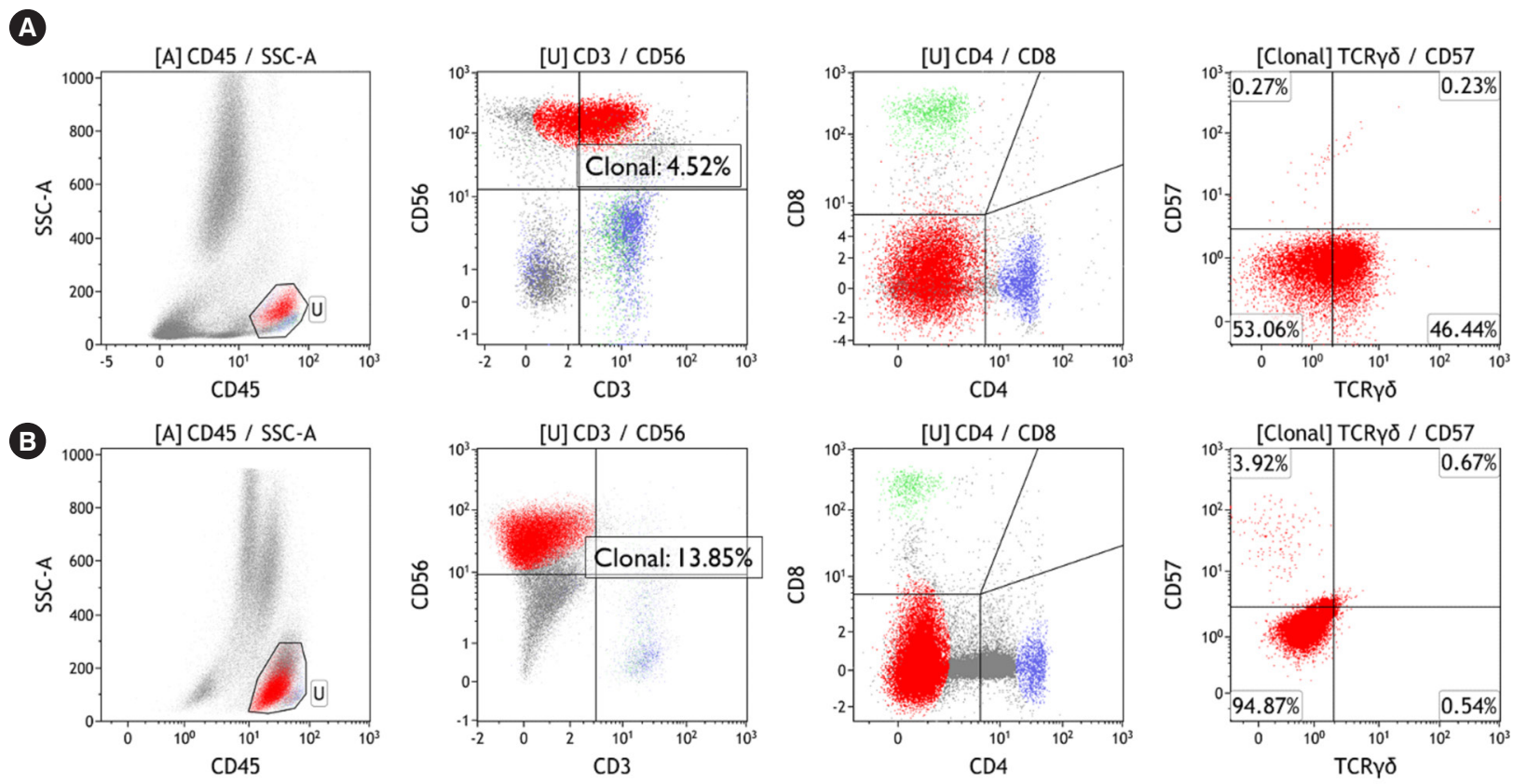

Fig. 1. Flow cytometry findings in both $(A)$ initial and $(B)$ follow-up studies. $(A)$ Abnormal lymphoid cells highlighted in red exhibited the sCD3+, CD4-, CD8-, and TCR $\gamma \delta+$ phenotype at diagnosis, (B) whereas they exhibited the sCD3-, CD4-, CD8-, and TCR $\gamma \delta-$ phenotype at relapse. The cells highlighted in blue and green are normal CD4+ T cells and CD8+ T cells, respectively. The CD4dim+ population observed at relapse (B) was probably made up of monocytes.

Abbreviations: BM, bone marrow; TCR, T-cell receptor.

Table 1. Reported cases of HSTL with loss of sCD3 and TCR $\gamma \delta$ at relapse

\begin{tabular}{|c|c|c|c|c|c|c|c|c|c|c|c|}
\hline \multirow{2}{*}{$\begin{array}{l}\text { Case } \\
\text { number }\end{array}$} & \multirow{2}{*}{\multicolumn{2}{|c|}{ Reference }} & \multicolumn{5}{|c|}{ Immunophenotypes } & \multirow{2}{*}{ Chromosome } & \multirow{2}{*}{$\begin{array}{c}T C R \\
\text { rearrangement }\end{array}$} & \multirow{2}{*}{ Therapy } & \multirow{2}{*}{ Outcome } \\
\hline & & & sCD3 & cCD3 & TCR $\gamma \delta$ & CD16 & CD56 & & & & \\
\hline 1 & $\begin{array}{l}\text { Farcet, et al. } \\
(1990) \text { [3] }\end{array}$ & Initial & + & NA & + & - & - & NA & + & ANT-based + CYC & Expired \\
\hline \multirow[t]{2}{*}{2} & \multirow{2}{*}{$\begin{array}{c}\text { Hodkoff, et al. } \\
\text { (2018) [6] }\end{array}$} & Initial & + & NA & + & - & - & $i(7)(q 10),+8$ & + & $\mathrm{CHOP}$ & Expired \\
\hline & & F/U after 2 months & - & - & - & Partial+ & - & $\mathrm{i}(7)(\mathrm{q} 10),+8$ & + & & \\
\hline 3 & Present case & F/U after 6 months & - & + & - & + & + & Complex w/i(7)(q10) & + & & \\
\hline
\end{tabular}

Abbreviations: HSTL, hepatosplenic T-cell lymphoma; F/U, follow-up; NA, not available; ANT, anthracycline; CYC, cyclophosphamide; CHOP, cyclophosphamide, hydroxydaunorubicin (doxorubicin), oncovin (vincristine), prednisolone; ICED, ifosfamide, carboplatin, etoposide, dexamethasone; GDP, gemcitabine, dexamethasone, cisplatin; SMILE, dexamethasone, methotrexate, ifosfamide, I-asparaginase, etoposide; TCR, T-cell receptor. 
gested that HSTL tends to gain an extra isochromosome 7q during the course of the disease, which may promote the proliferation of abnormal cells [7]. The patient expired two months after relapse.

There are only two reports on the loss of sCD3 and TCR $\gamma \delta$ in HSTL during follow-up [3, 6]. Detailed information regarding each case is presented in Table 1.

Compared with previous cases, determining disease relapse was very challenging in the present case because of CD56 positivity and simultaneous loss of SCD3 and TCR $\gamma \delta$ expression, which made the abnormal lymphoid cells from this case difficult to distinguish from reactive NK cells. In such cases, a meticulous review of cell morphology, cytogenetic studies, and molecular studies should be conducted. Morphologically, immunohistochemistry for granzyme B and perforin could be useful, as these proteins are not expressed in HSTL but are expressed in aggressive NK-cell leukemia (ANKL) $[2,4]$. Conversely, HSTL cases could be positive for TIA-1 expression [2, 4]. Identification of isochromosome $7 q$ and $T C R$ rearrangement may also aid the differential diagnosis of HSTL versus ANKL $[2,4]$. Due to the limited number of studies, the clinical significance of sCD3 and TCR $\gamma \delta$ loss remains to be elucidated. However, one report suggested that sCD3 and TCR $\gamma \delta$ loss are associated with poor prognosis [3].

While flow cytometry provides powerful insights into neoplastic cell lineage, it may be a double-edged sword in cases where lineage-specific markers are aberrantly lost, since it could lead to the misclassification of cell lineages. Hematopathologists should always consider the possibility of unusual immunophenotypic changes. Since only a few such cases have been reported to date, the fact that HSTL can present without expression of SCD3 and TCR $\gamma \delta$ is not well known. Hence, we present this diagnostically challenging case to share our experience and highlight the fact that T-lineage lymphoma cells with loss of T-cell markers could be mistakenly identified as NK cells in flow cytometry.

\section{ACKNOWLEDGEMENTS}

We would like to acknowledge Sung Hoan Bang, M.T. for conducting the flow cytometry.

\section{AUTHOR CONTRIBUTIONS}

Lee B wrote the manuscript. All authors contributed to data interpretation. Kim HY and Cho D critically reviewed the manu- script. All authors have read and approved the final version of the manuscript.

\section{CONFLICTS OF INTEREST}

There are no potential conflicts of interest relevant to this article to report.

\section{RESEARCH FUNDING}

None declared.

\section{ORCID}

Beomki Lee https://orcid.org/0000-0003-3463-2121

Dae Jin Lim https://orcid.org/0000-0003-3079-5716 Won Young Heo Sang Eun Yoon https://orcid.org/0000-0003-2695-9634 https://orcid.org/0000-0002-0379-5297 Hyun-Young Kim Hee-Jin Kim https://orcid.org/0000-0003-0553-7096 https://orcid.org/0000-0003-3741-4613 https://orcid.org/0000-0002-7542-5551 https://orcid.org/0000-0001-6861-3282

\section{REFERENCES}

1. Ferreri AJ, Govi S, Pileri SA. Hepatosplenic gamma-delta T-cell lymphoma. Crit Rev Oncol Hematol 2012;83:283-92.

2. Gaulard P, Jaffe ES, Krenacs L, Macon WR. Hepatosplenic T-cell lymphoma. In: Swerdlow SH, Campo E, et al. eds. WHO classification of tumours of haematopoietic and lymphoid tissues. Revised 4th ed. Lyon: IARC, 2017:381-2.

3. Farcet JP, Gaulard P, Marolleau JP, Le Couedic JP, Henni T, Gourdin MF, et al. Hepatosplenic T-cell lymphoma: sinusal/sinusoidal localization of malignant cells expressing the T-cell receptor gamma delta. Blood 1990;75:2213-9.

4. Kapur LH, Khaled Y, Solh M, Ward D, Chang CC. De novo CD3 negative hepatosplenic T-cell lymphoma: diagnostic challenges and pitfalls. Arch Pathol Lab Med 2014;138:969-73.

5. Chauhan R, Tyagi S, Mirgh S, Mishra P, Seth T, Mahapatra M, et al. Expect the unexpected-Loss of surface CD3 on flow cytometry in hepatosplenic T-cell lymphoma: an eye opener. Indian J Pathol Microbiol 2018;61:275-7.

6. Hodkoff A, Bentley P, Xu X. Loss of CD3 and TCR $\gamma \delta$ expression in a relapsed hepatosplenic T-cell lymphoma. Leuk Lymphoma 2018;59:47981.

7. Wlodarska I, Martin-Garcia N, Achten R, De Wolf-Peeters C, Pauwels P, Tulliez $\mathrm{M}$, et al. Fluorescence in situ hybridization study of chromosome 7 aberrations in hepatosplenic T-cell lymphoma: isochromosome 7q as a common abnormality accumulating in forms with features of cytologic progression. Genes Chromosomes Cancer 2002;33:243-51. 